\title{
A Study on Intelligent Manufacturing Industrial Internet for Injection Molding Industry Based on Digital Twin
}

\author{
Zhiyong Wang $\mathbb{D},,^{1,2,3}$ Wei Feng $\mathbb{D}^{1,4,5}$ Junlin Ye $\mathbb{D}^{1,2,3}$ Jinbiao Yang $\mathbb{D}^{6}$, and Chun Liu $\mathbb{D}^{6}$ \\ ${ }^{1}$ Shenzhen Institutes of Advanced Technology, Chinese Academy of Sciences, Shenzhen, China \\ ${ }^{2}$ Guangdong Provincial Key Lab of Robotics and Intelligent System, Shenzhen Institutes of Advanced Technology, \\ Chinese Academy of Sciences, Shenzhen, China \\ ${ }^{3}$ CAS Key Laboratory of Human-Machine Intelligence-Synergy Systems, Shenzhen Institutes of Advanced Technology, \\ Shenzhen, China \\ ${ }^{4}$ University of Chinese Academy of Sciences, Beijing, China \\ ${ }^{5}$ The Chinese University of Hong Kong, Hong Kong, China \\ ${ }^{6}$ Shenzhen Zk-Cenda Connected Intelligent Technology Co. Ltd., Shenzhen, China \\ Correspondence should be addressed to Wei Feng; wei.feng@siat.ac.cn
}

Received 6 August 2020; Revised 9 September 2020; Accepted 17 December 2020; Published 13 January 2021

Academic Editor: Dan Selisteanu

Copyright $\odot 2021$ Zhiyong Wang et al. This is an open access article distributed under the Creative Commons Attribution License, which permits unrestricted use, distribution, and reproduction in any medium, provided the original work is properly cited.

\begin{abstract}
As one of the basic manufacturing industries in China, injection molding industry is faced with the problems of low degree of informatization and intelligence, resulting in low production efficiency and high costs. It is urgent to integrate deeply with new generation of information technology to achieve transformation and upgrade. In this paper, an integrative industrial Internet architecture of "integration of intelligent equipment, intelligent production lines, intelligent workshops, intelligent factories, and intelligent formats" was described. The injection molding intelligent control system, the production management, control platform based on the MES system, and other key technologies were researched. Also, the smart factory architecture based on digital twin was established, and the implementation method of the smart factory digital twin system was elaborated. The feasibility and effectiveness of the method had been verified through the industrial application, which provided the technical supports for the injection molding industrial Internet system. Finally, the intelligent manufacturing industrial Internet cloud platform for injection molding industry was prospected.
\end{abstract}

\section{Introduction}

In recent years, China's injection molding industry has developed rapidly and has become the world's largest producer and consumer of plastic products, which plays an important role in the national economic and social development [1]. However, the overall technical level of China's injection molding industry still has a large gap with the developed countries, especially in the low degree of development of equipment intelligence, production intelligence, management intelligence, and service intelligence, resulting in low production efficiency, high costs, serious waste, and other issues. This situation severely restricts the development and transformation of China's injection molding industry to high efficiency, low cost, integration, precision, and environmental protection $[2,3]$. With the development of national strategies such as "Made in China 2025" and "New Infrastructure Construction," deep integration of manufacturing and new generation of information technology have become the keys of innovation and development for China's manufacturing [4]. In this paper, an industrial Internet architecture for injection molding industry was described based on the current situation of China through the digitization, networking, and intelligence technical method. The smart factory system for injection molding based on digital twin was established. Then, the injection molding intelligent control system, production management, and control platform based on the MES 
system and other key technologies were researched. At last, the intelligent manufacturing ecosystem of "intelligent equipment, intelligent production line, intelligent workshop, intelligent factory, and intelligent format" for injection molding industry was designed and implemented.

\section{Architecture of the Industrial Internet Platform for Injection Molding Industry}

2.1. Industrial Internet. Industrial Internet has become a new type of industrial manufacturing and service system and connected with all elements, industry chains, and value chains through the full interconnection of personnel, machines, materials, process, and environment in the production process. It has become the infrastructure supporting the fourth industrial revolution and intelligent manufacturing [5-7]. The essence of industrial Internet is the triple integration of Information Technology (IT), Communication Technology (CT), and Operational Technology (OT) [8-10]. It can connect all kinds of internal and external data of products, equipment, production lines, factories, suppliers, customers, and other enterprises and realize interconnection and interoperability across devices, systems, factories, and regions through CT. It can also mine and analyse massive industrial data based on IT and make the invisible processes and experiences explicit, digital, reusable, and predictable in the traditional manufacturing process combined with OT. Finally, industrial Internet forms the precipitation of industrial experience and mechanism models, promotes the transformation of intelligent control, operation optimization, and production organization, and realizes the intelligence of the entire manufacturing service system.

\subsection{Value Analysis of Digital Transformation for Injection} Molding Industry. A double-helix model based on three perspectives and nine dimensions is used to analyse the digital transformation value for injection molding industry (see Figure 1). The deep integration application of industrial Internet takes the industry value reconstruction as the main line and adopts two-wheel-driven and two-way-iteration of technology and business to realize the business-value and business-technology closed loop. In the value view, the industrial Internet platform connects and reconstructs all elements, industrial chains, and value chains of injection molding industry, which finally achieves quality improvement and efficiency, cost savings, and ecological cultivation. In the technical view, industrial big data is collected, transmitted, calculated, analysed, and applied through the key technologies of edge computing, artificial intelligence, and digital twin. In the business view, the industrial Internet platform can realize full-cycle control of materials, equipment, components, and products, excavate the pain points of intelligent upgrading at the equipment level, workshop level, enterprise level, and industry chain level, and achieve a gradual transition from the application of a single device or scene to a complete production system or management process. Therefore, it finally evolved to coordinate industrial resources to realize the intelligent production, network collaboration, personalized customization, and service extension of the industry.

2.3. Architecture of the Industrial Internet Platform for Injection Molding Industry. Injection molding industry is a kind of discrete industry with multivariety, small-batch production, and complicated process. The single-machine automation and rigid production lines were mainly used, while the flexible production lines were few. The OT system and IT system had difficulty in achieving interconnection. Data collection was very difficult and lacked a complete dataintegration application framework because there were many types and brands of production equipment and also lacked open interfaces and unified interconnection standards. Therefore, combined with the value analysis and characteristics of digital transformation for injection molding industry, the architecture of the industrial Internet platform was built as follows (see Figure 2):

(1) Intelligent perception and control layer: firstly, the controller, sensor, and collector were used to realize the intelligent perception of injection molding process such as injection pressure, injection speed, barrel temperature, and machine speed. Through industrial data real-time collection and edge calculation of key equipment such as injection molding machine, mold and $\mathrm{CNC}$ machine, and the intelligent setting and optimization of key process parameters of injection molding process based on the data-driven, as well as the application of industrial auxiliary production robots and unmanned production line, and the equipment and production line could be made intelligent.

(2) Network connection layer: all elements of injection molding production and manufacturing environment were interconnected by building cloud servers, data centres, cloud computing centres, and other infrastructures.

(3) Cloud management platform layer: through the cloud computing architecture and platform of human-machine integration, focusing on the full life-cycle management process of products and equipment, integrated management, and optimization of design, production, service, and other data was implemented in the cloud management platform.

(4) Data analysis layer: the collected industrial big data was integrated and analysed. The production process was optimized through the analysis and feedback of real-time production data in the industrial site management. The scientific decisions were formed based on big data mining and analysis in the business operation. At last, it realized hierarchical informatization, automation-intelligent management, and intelligent control for factories.

(5) Application service layer: industrial Internet and industrial big data of injection molding enterprises and their upstream and downstream supporting 

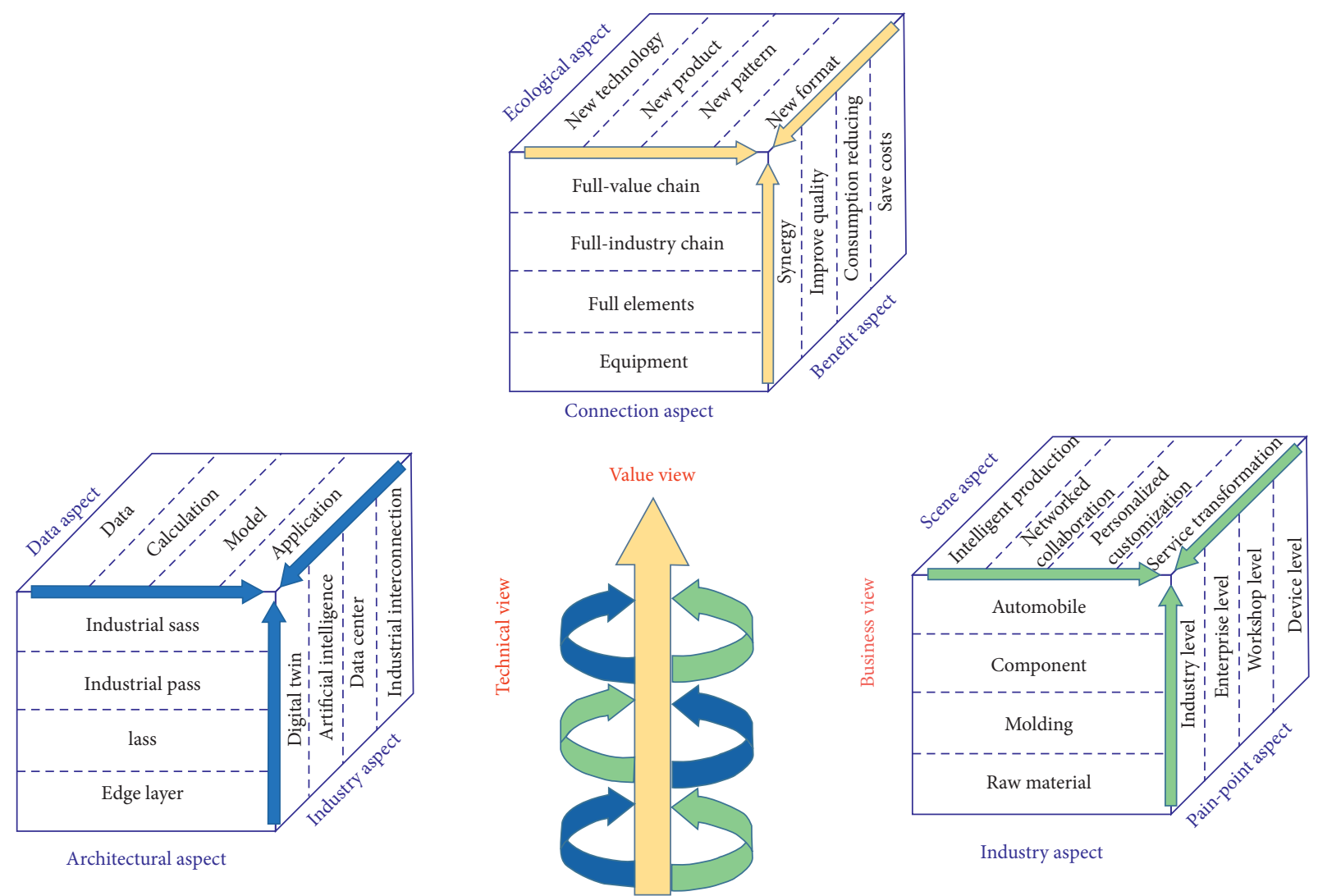

FIGURE 1: The digital transformation framework for injection molding industry based on the double-helix model of three perspectives and nine dimensions.

enterprises were established based on the above situation. Then, the cloud platform for injection molding industry through collaborative sharing of information resources was achieved. It can carry out intelligent application services such as order adjustment, supply chain transactions, design collaboration, talent training, and technical services and promote to construct an intelligent format for injection molding industry finally.

\section{Architecture of Injection Molding Smart Factory Based on Digital Twin}

According to the architecture of the industrial Internet platform, the enterprise-level smart factory must be constructed to promote the intelligent format of injection molding industry. Digital twin could effectively contribute the interconnection and deep integration of physical space and information space for the factory and enhance the intelligent level of injection molding production and management.

3.1. Digital Twin Definition. Digital twin, using information technologies comprehensively such as perception, modeling, and calculation, builds a high-fidelity model in the virtual space that is consistent with the physical space in the digital form. Through the information real-time interaction feedback and data fusion in the physical space, it can simulate the behaviors of objects, monitor, diagnose, predict, and make decisions for the physical space and realize the interactive mapping between the physical space and virtual space [11]. Because digital twin has the characteristics of virtual real integration and real-time interaction, iterative operation and optimization, and full element/process/ business data-driven, it is widely used in the whole life-cycle process of product development, design, manufacturing, operation, maintenance, and service. It has become the key enabling technology which supports the digital transformation for manufacturing industry, and it is also the core for industrial Internet landing practice.

3.2. Digital Twin Model. The digital twin model for injection molding industrial application must be created first. Combined with Professor Tao Fei's five-dimension digital twin model [12-14], the digital twin model of injection molding industry was shown (see Figure 3). The physical entities are the foundation and mainly include the unit-level and system-level physical entities such as injection molding machines, molds, CNC machines, production lines, workshops, and enterprises. The virtual entities are digital models of these physical entities, which can describe the above physical entities with high fidelity, multiple time scales, and multiple spatial dimensions. The services are reconstruction values of digital twin. Through the processing and analysis of 


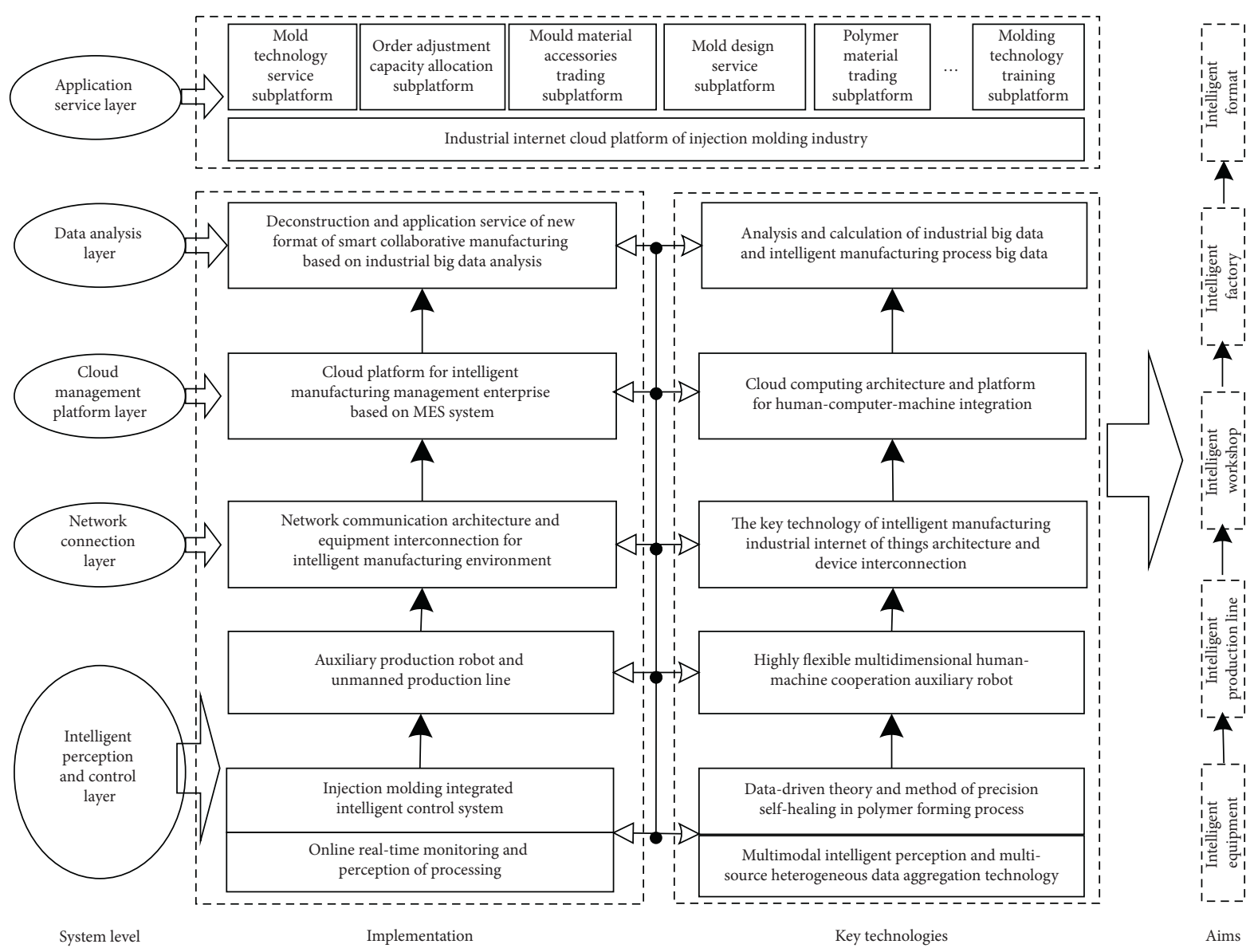

FIgURE 2: Architecture of the industrial Internet platform for injection molding industry.

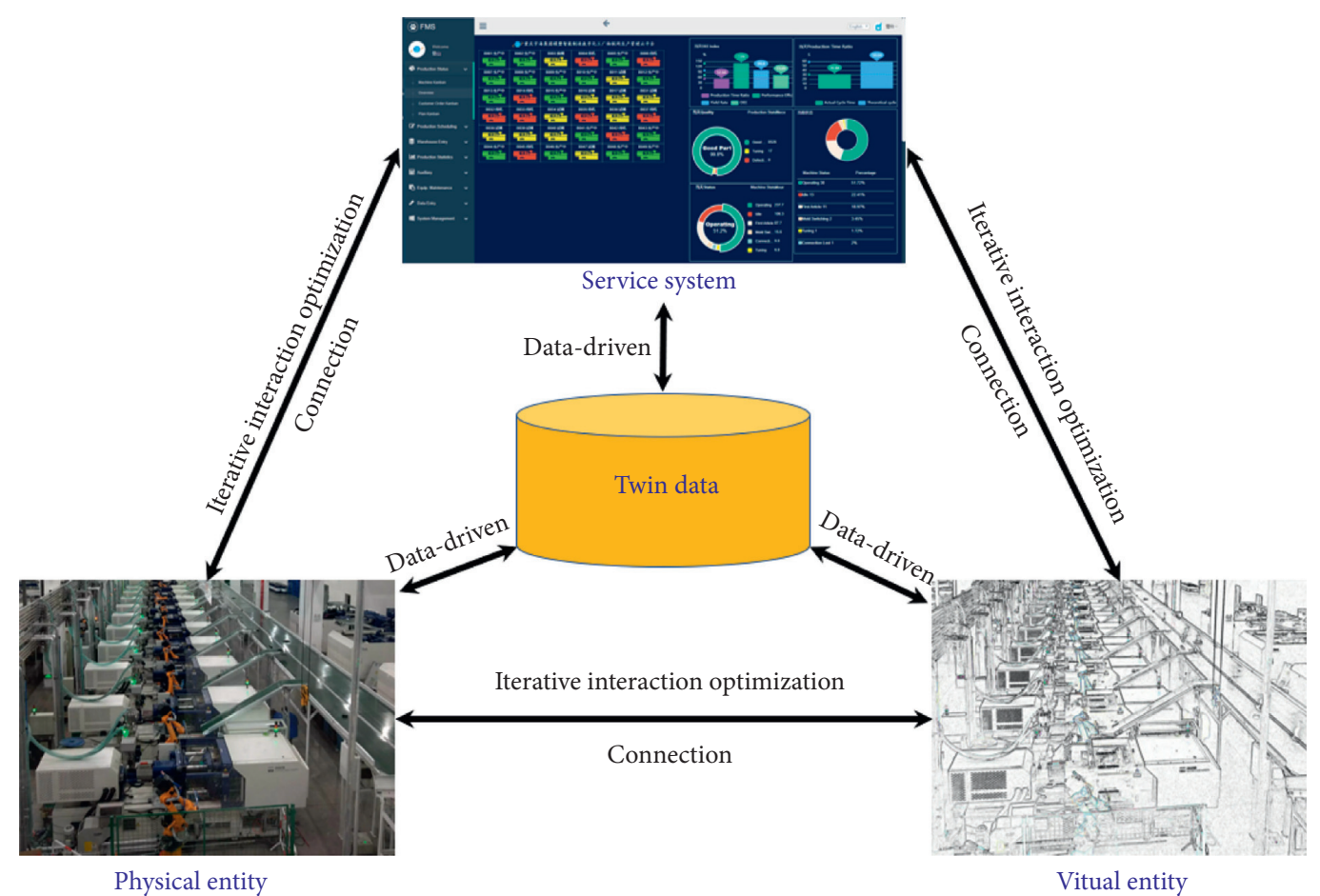

Figure 3: The five-dimension digital twin model. 
industrial big data, it can provide functions such as intelligent setting and optimization of process parameters, process monitoring and tracing, production scheduling and collaboration, equipment operation, and maintenance and finally make scientific decisions. Digital twin data is the driver of digital twin technology, mainly including physical entity data, virtual entity data, service data, and knowledge data, and, at the same time, realizes interconnection and iterative interaction optimization between the object entities, virtual entities, and services [15-20].

3.3. Architecture of Smart Factory for Injection Molding. According to the digital twin model, the architecture smart factory for injection molding was built (see Figure 4).

(1) Data layer: digital twin data is the foundation. Through the multisource heterogeneous sensing and multiprotocol compatible real-time collection of industrial data with personnel, products, materials, equipment, process, and environment, the virtual space is established which could map the physical space of all elements such as equipment, production lines, workshops, and enterprises. The industrial data is the key to realize injection molding process in the physical space.

(2) Model layer: the digital twin model is the core, which is the digital reconstruction of the production activities of the entire process, and realizes virtual reproduction, analysis, optimization, and decisionmaking functions of injection molding production activities in the virtual space.

(3) Application layer: the applications are the purpose. Real-time monitoring for the user can achieve through the real-time mapping for the production activities. The optimization and scientific decision-making of the process can be realized through processing and analysis of the industrial data of interactive mapping, which promotes a two-way dynamic interaction between the physical space and virtual space. The full lifecycle application services from product design, mold design, mold manufacturing, and injection production to industrial collaboration will be achieved.

\section{Implementation of the Digital Twin System for Injection Molding Smart Factory}

In order to realize the functions of application layer, the modeling of full-factor physical space, data acquisition, and real-time mapping in the production activities are the key aspects of the digital twin system for injection molding smart factory $[14,21-26]$.

4.1. Digital Twin Modeling of All Elements in Physical Space. Because there are different physical entity types, diversified functions, and entity-generated data, it is needed to build a unified logical structure for digital twin modeling:
$D T_{\text {mi }}=D T_{\text {pers }} \mathrm{U} D T_{\text {mat }} \mathrm{U} D T_{\text {mold }} \mathrm{U} D T_{\text {machine }} \mathrm{U} D T_{\text {env }}$.

In formula (1),

(1) $D T_{\mathrm{mi}}$ is the digital twin model of the production process.

(2) $D T_{\text {pers }}$ is the digital twin model of personnel, which is mainly reflected in the movement and spatial position. The human body structure is mapped through the three-dimensional structure model, and the identification and position positioning are performed by RFID or fingerprint image recognition. The position and identity of personnel is controlled in the digital space.

(3) $D T_{\text {mat }}$ is the digital twin model of materials, which is mainly the spatial location and inventory status of plastic raw materials, mold parts, tools, etc. It can obtain material data and realize material management through the warehousing material management system or ERP system.

(4) $D T_{\text {molt }}$ is the digital twin model of the mold, which is mainly the CAD/CAM/CAE/CAPP data, and can be directly obtained through the PDM system or CAX system. The modifications or changes of the mold can be interacted in real time.

(5) $D T_{\text {machine }}$ is the digital twin model of devices, which is the most critical and complex. The devices of injection molding industry are mainly used for mold processing and inspection, injection production and assembly, etc., including CNC machines, coordinate measuring instruments, injection molding machines, industrial robots, AGV, and auxiliary equipment. In order to complete the true mapping of the digital twin model to the physical entities, the model must ensure that the three-dimensional size and behavior are highly consistent with the physical entities of these devices. It is necessary to establish a virtual and real communication control interface to obtain data in real time and define related virtual service to complete the device's action and behavior.

(6) $D T_{\text {env }}$ is the digital twin model of environment, which is mainly the digital model of physical space environment.

4.2. Real-Time Data Collection. During the production process of injection molding, the industrial data is generated. There are a lot of different types, brands, and models of devices on the production site, and their interface protocols are also different. In order to solve the problem of heterogeneous equipment data collection and virtual real interactive fusion, it is necessary to establish a unified standardized communication framework and protocol. The OPC UA protocol supports complex built-in data and crossplatform operation and provides unified address space and services [27-30]. Most of the above devices support the OPC UA protocol, so communication architecture of the digital twin based on the OPC UA protocol for injection molding production process was adopted (see Figure 5). 


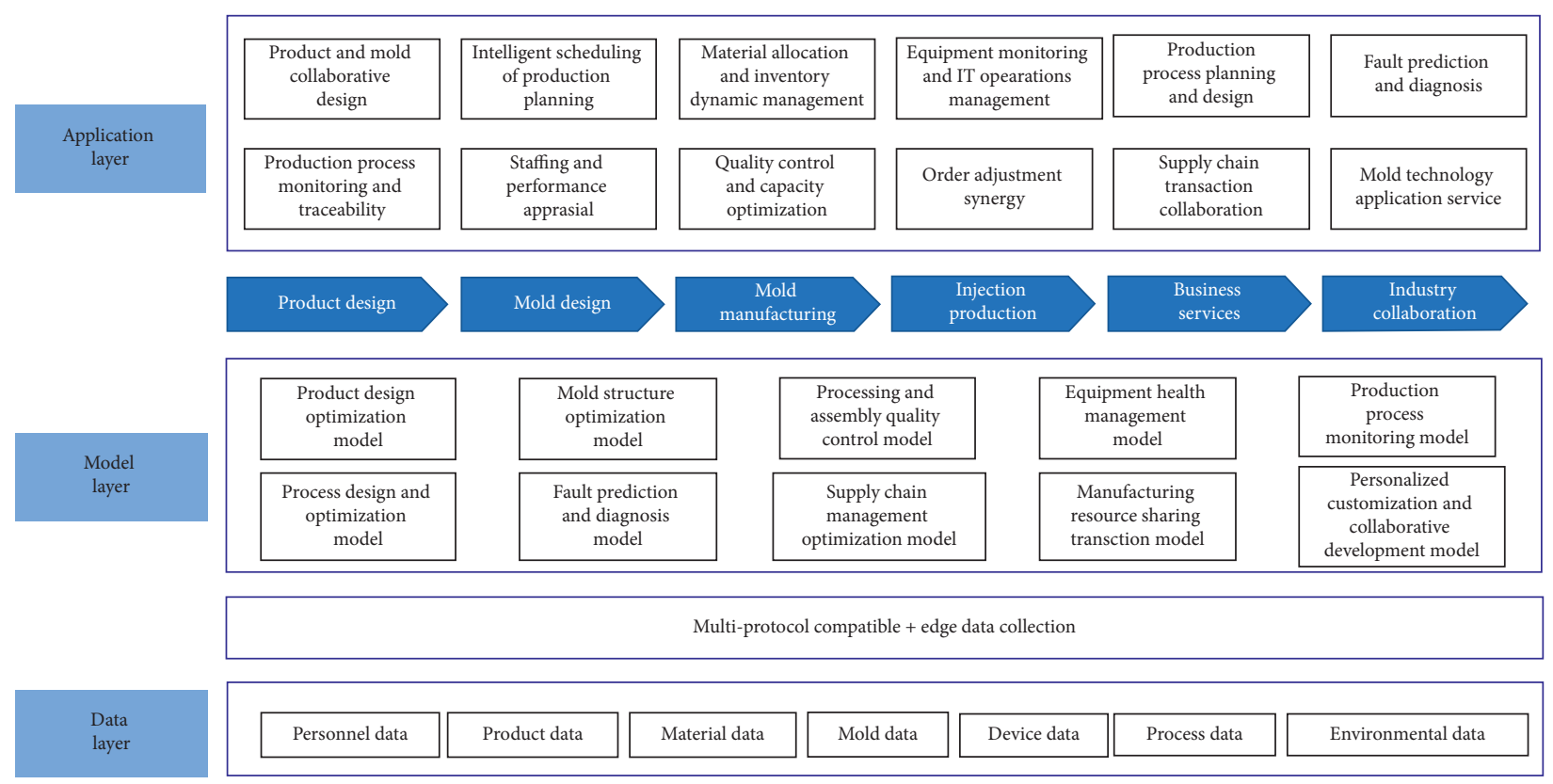

FIgURE 4: Architecture of injection molding smart factory based on digital twin.
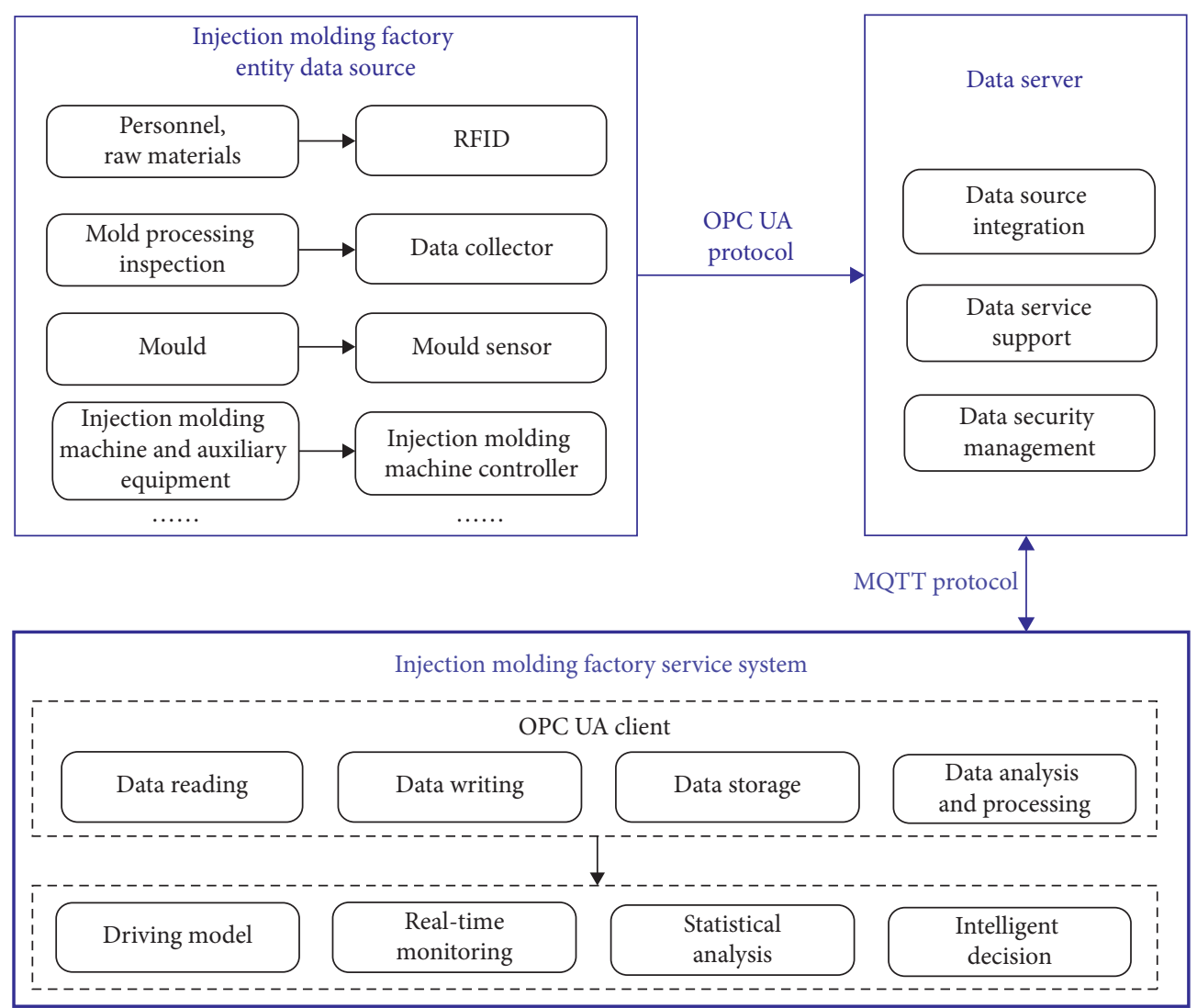

FIGURE 5: Communication architecture of digital twin based on the OPC UA protocol for the injection molding production process.

In the communication architecture, the underlying data of the production process was obtained through RFID, data collectors, mold sensors, injection molding machine controllers, etc. Based on the OPC UA protocol, the collected industrial production data was transmitted to the data server in real time by industrial fieldbus or ethernet. After the data server aggregated the field data and device information, it was converted into the data which supported the OPC UA 
protocol and provided the corresponding services for the clients after data management and logical operations. As a client of OPC UA, the molding plant service system obtained the corresponding real-time data from the server for data reading and writing, storage, and analysis processing. On this condition, it could drive various element models, update, and monitor various elements in real time and further perform statistical analysis and intelligent decision-making.

\subsection{Real-Time Interactive Mapping and Operation} Mechanism. The interactive mapping and operation mechanism of the digital twin system for injection molding factory is shown (see Figure 6).

(1) Iterative optimization of production factor management: when the injection molding factory received the order task, the factory service system was driven by historical data of production factor management in the factory twin database and performed initial configuration according to the production task of the current order. The factory service system obtained the real-time data of production factors such as personnel, molds, injection molding machines, and materials of the physical factory. By analysing, evaluating, and predicting the status of these production factors, the system optimized the initial configuration plan, generated order control instructions, and guided the physical factory to allocate production factors. The physical factory transmitted the data into the factory service system for real-time prediction and optimization during the process of allocating production factors until the optimal configuration of production factor management for the production order was achieved and production planning tasks were obtained. The data generated during the iterative optimization of production factor management was transmitted and saved in the factory twin database, which could be used for the foundation and drive of data in the subsequent stage.

(2) Iterative optimization of automatic scheduling of production planning: after the virtual factory received the production plan, driven by historical simulation data in the factory twin database and realtime data of physical factory, the factory service system simulated and optimized the production plan schedule based on the logic and rule model of optimal time and the lowest cost of production and fed back optimization results of automatic schedule to the factory service system. Then, the system optimized these results and transmitted them to the virtual factory and iteratively optimized until the best plan for the production planning schedule and the production operation instruction based on the plan were obtained. The data generated in the iterative optimization of the automatic scheduling of production plan was also transmitted and saved in the factory twin database, which could be used for the foundation and drive of data in the subsequent stage.
(3) Real-time monitoring and iterative optimization of production process: the physical factory received the production operation instructions of the previous stage to organize production. In the actual production process, the physical factory transmitted the production process data to the virtual factory in real time and the virtual factory also monitored the physical factory's production process in real time. When an abnormal production situation occurred, the virtual factory performed production scheduling simulation and comprehensively evaluated, predicted, and optimized the digital twin data based on the simulation results, real-time production data, and historical production data. It generated real-time production scheduling instructions to guide the physical factory's scheduling. The data generated in this process was also transmitted and saved in the factory twin database, which could be used for the foundation and drive of data in the subsequent stage.

(4) Iterative simulation of fault prediction, diagnosis, and capacity prediction: during the production process, the physical factory transmitted the production process data to the virtual factory in real time. Then, the virtual factory performed fault prediction, diagnosis simulation, capacity prediction, etc. and made assessments and predictions according to the simulation results, real-time production data, and historical production data. The real-time monitoring instructions were generated to realize equipment maintenance, production capacity prediction, and evaluation. The iterative optimization of production factor management was driven by the instructions in the iterative simulation of capacity prediction. The data generated in this process was also transmitted and saved in the factory twin database, which could be used for the data foundation and driving of the subsequent stage.

\section{Key Technologies}

5.1. Injection Molding Integration Intelligent Control Technology. Injection molding is a kind of typical intermittent industrial production process. The molten plastic materials are made into a certain shape of plastic products through pressurization, injection, cooling, and separation. The quality of plastic products depends on material properties, injection machine variables, molding process, and interaction of these parameters. The injection molding machine and plastic mold are the key equipment. As the "brain" of the injection molding machine, the control system plays an important role in the repeatability and stability of the process. The state of the plastic melt in the mold cavity also affects product quality and production efficiency directly. Therefore, in order to realize the intelligent perception of injection molding process, the parameters of the injection molding machine and status data of plastic mold must be collected. At present, there are many brands of 


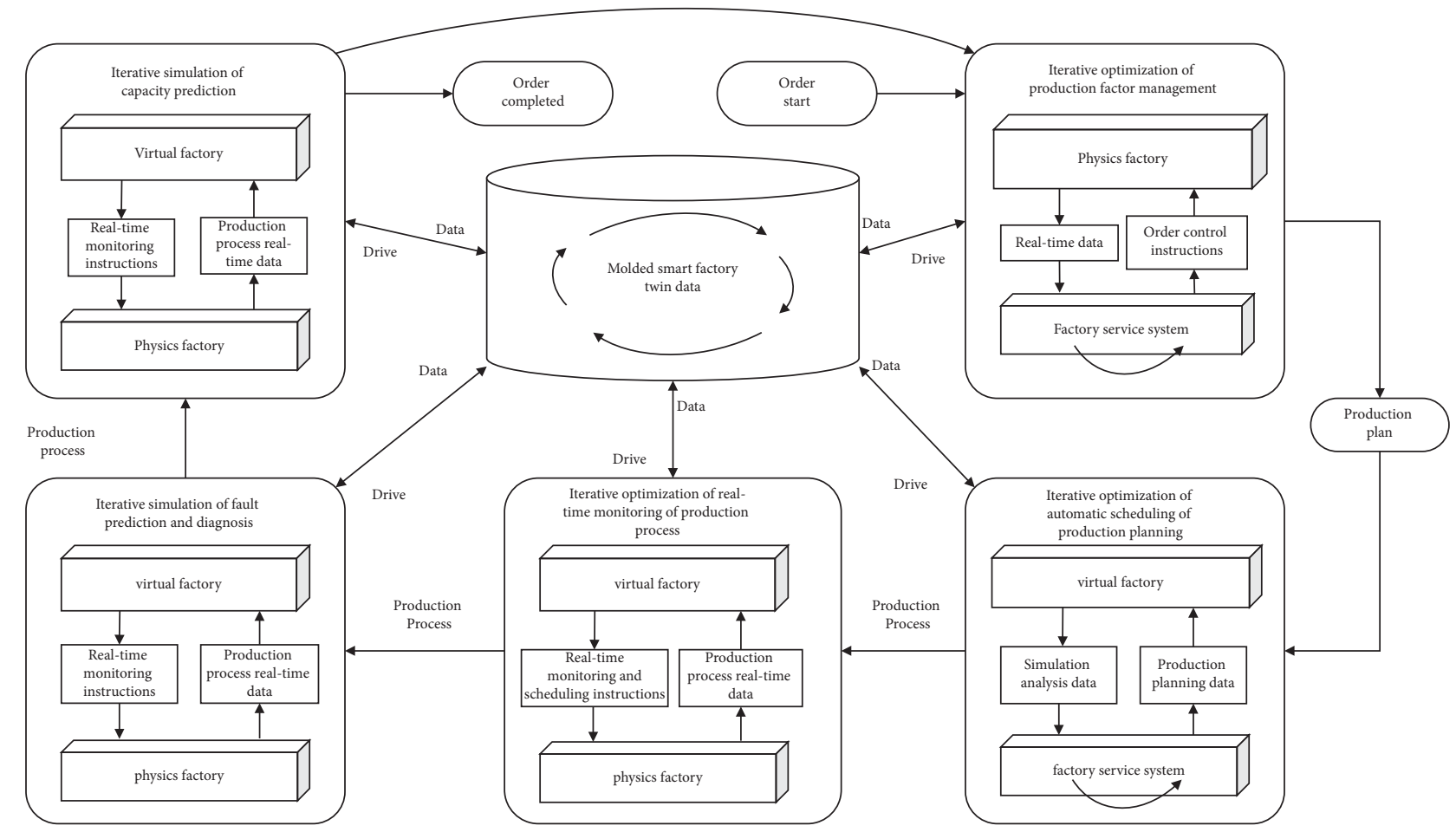

FIGURE 6: Real-time interactive mapping and operation mechanism of injection molding factory based on digital twin.

injection molding machines which lack open interfaces, and the function of the mold sensor is very single, which makes it difficult to achieve online perception of the whole injection molding process. Combined with the process characteristics of injection molding, as shown in Figure 7, the application of injection molding integrated intelligent control technology, including the high-precision injection molding machine control system and in-mold online quality perception system, could sense and monitor the parameters of the process and mold cavity plastic-melt state, respectively, and achieve intelligent setting and automatic optimization of the process parameters based on the data-driven. Finally, the real-time data collection and process quality closed-loop control of the whole process were achieved.

(1) The high-precision injection molding machine control system (see Figure 8): based on the EtherCAT architecture hardware platform with excellent scalability, compatibility, and unified data transmission interface, the control system could be applied to multiple brands of injection molding machines. Through two-dimensional time-iterative predictive control algorithm, it could achieve high-precision control of the key injection process parameters such as barrel temperature, injection speed, holding pressure, and mold opening position [31-36]. Combined with the current situation of high production, low value, and control accuracy of China's injection molding machine industry, without changing the mechanical mechanism of original machine, the control performance and repeatability of the original machine could be improved by only replacing the control system, and "precision injection" could be achieved.

(2) The in-mold online quality monitoring system based on capacitance measurement [37-39] (see Figure 9): during the various stages of injection molding, the change of plastic-melt state in the mold cavity caused the changes of its dielectric constant. Therefore, through the measurement and intelligent processing of the capacitance signal, the detection model is established to realize online monitoring of the flow state of plastic melt, detection of the weight of plastic product, and determination of optimal cooling time, so as to realize online closed-loop perception of the whole process.

(3) Through the integrated application of the above two systems, the fault diagnosis of the injection molding process was realized, and the quality model of injection molding process was established to optimize the process parameters based on the data-driven $[40,41]$, which could achieve "intelligent injection."

5.2. The MES System for Enterprise Cloud Platform Based on SaaS. The MES system of production management and control enterprise cloud platform based on SaaS (Softwareas-a-Service) $[42,43]$ (see Figure 10) was designed to achieve 


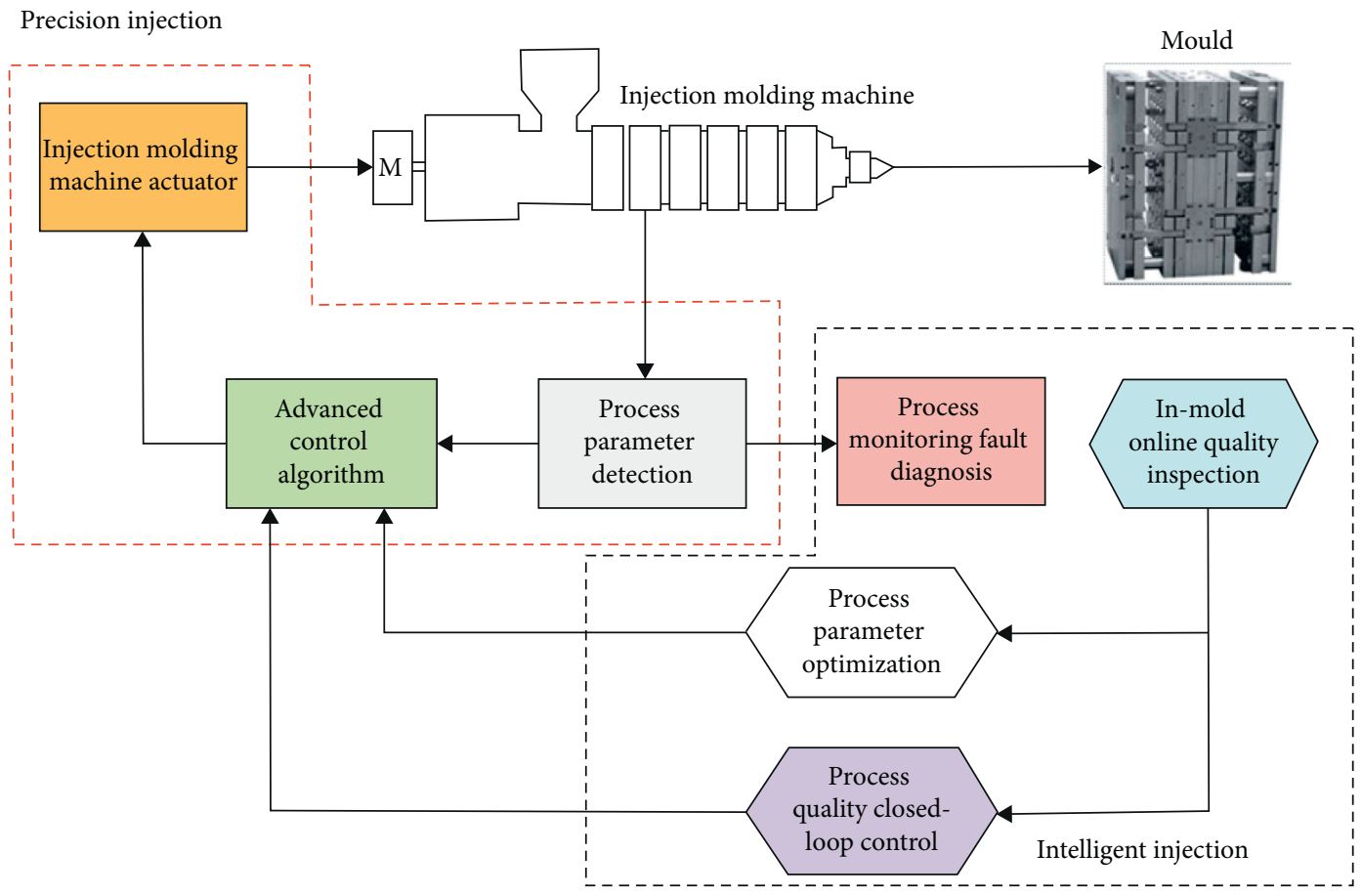

FIGURE 7: Schematic diagram of the injection molding integrated intelligent control system.

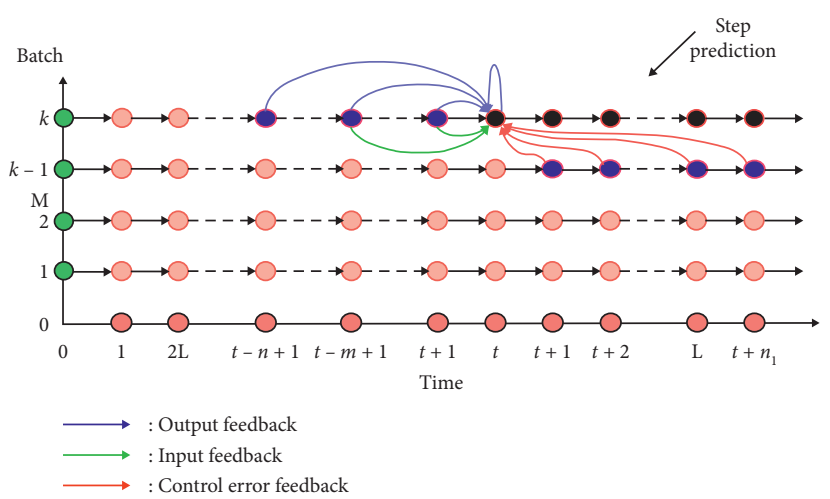

(a)

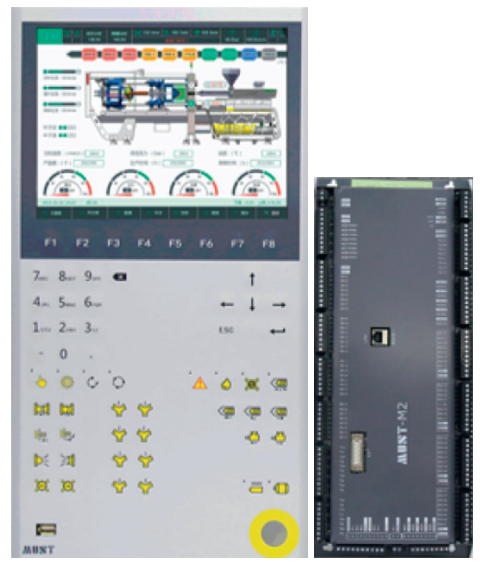

(c)

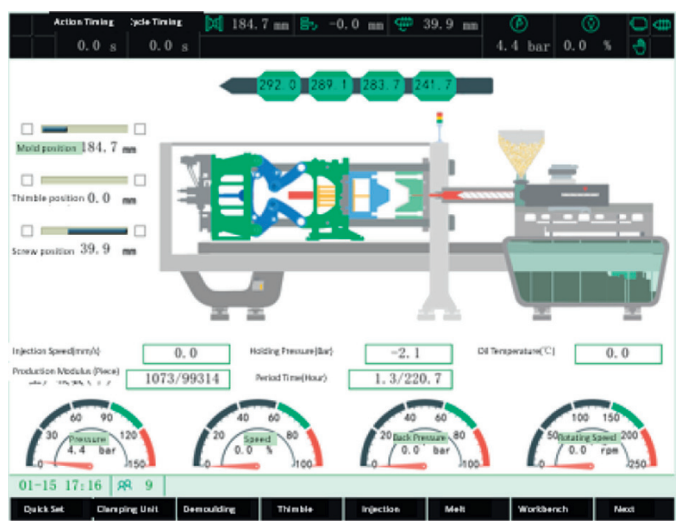

(b)

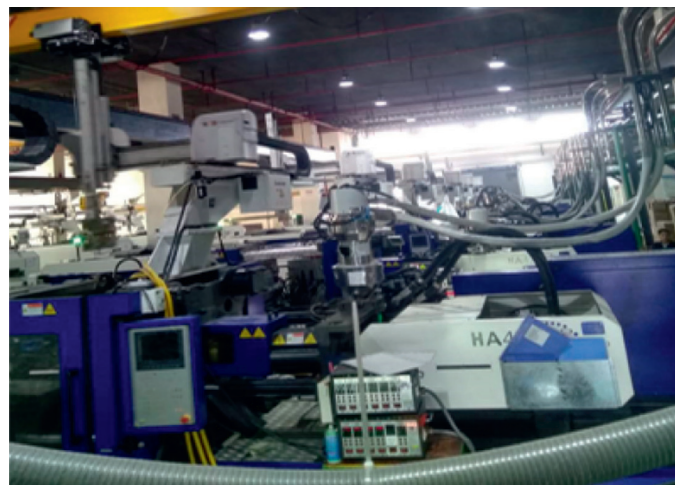

(d)

FIGURE 8: The high-precision injection molding control system and its application. (a) Schematic diagram of iterative predictive control algorithm based on two-bit time. (b) Software interface of the system. (c) Hardware platform of the system. (d) Industrial applications of the system. 


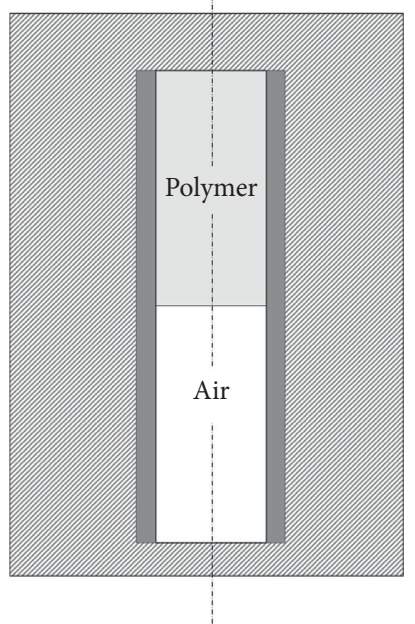

(a)

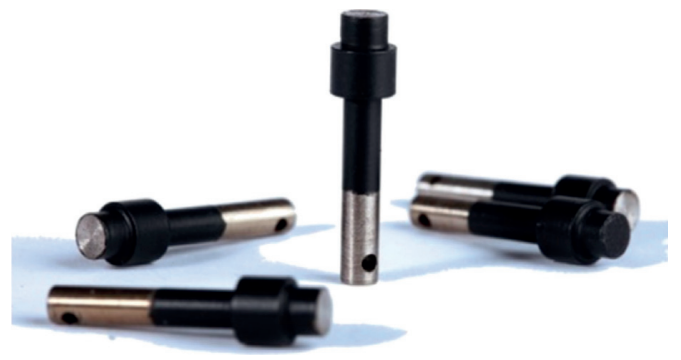

(c)

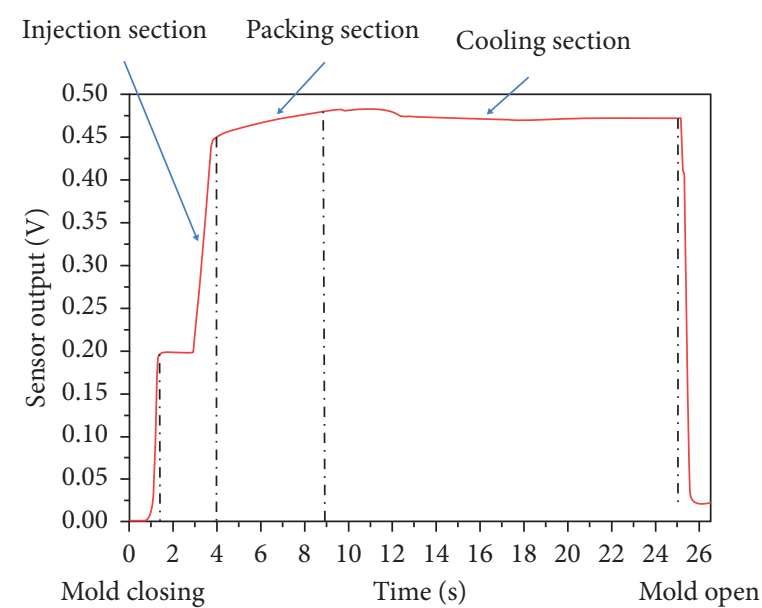

(b)

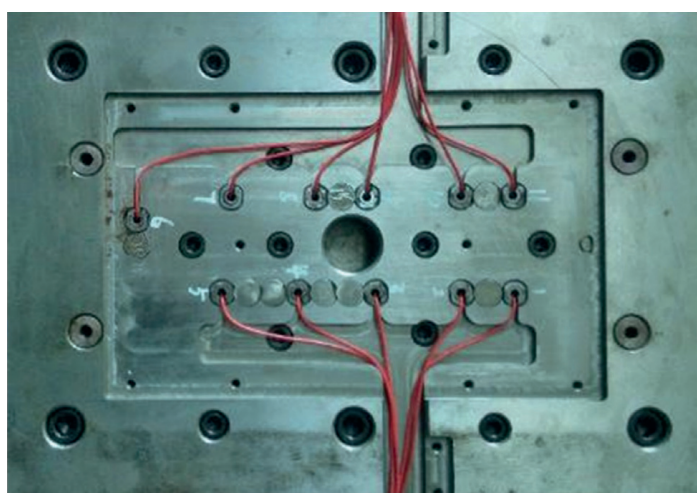

(d)

Figure 9: The online quality monitoring system and the application in the mold. (a) Illustration of the melt flow during the filling stage. (b) Capacitance measurement signal in the mold cavity during the whole injection molding process. (c) Sensor probe of the system. (d) Application of the system.

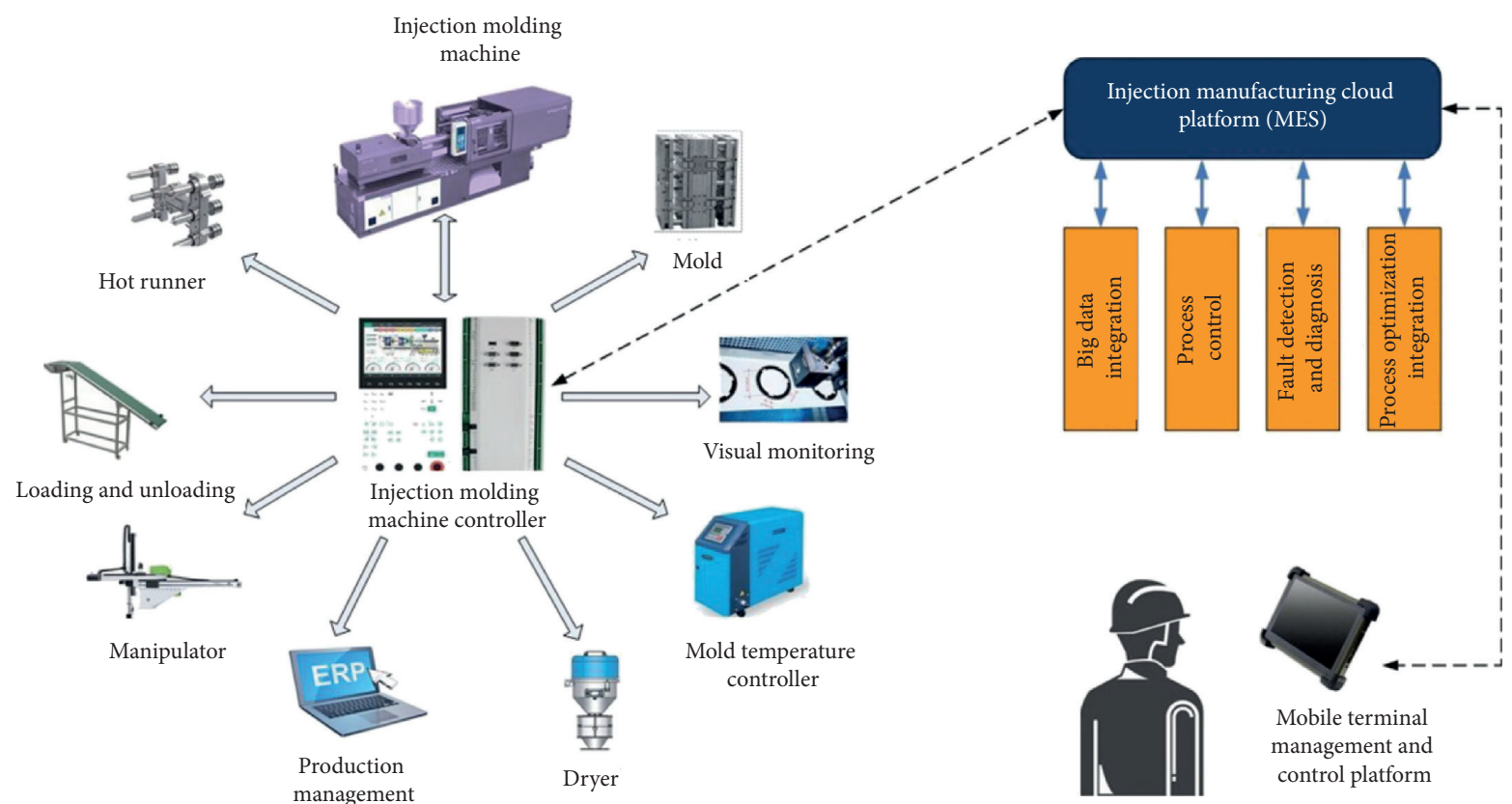

FIgURE 10: The MES system for the enterprise cloud platform based on SaaS. 
production planning and dynamic scheduling, equipment monitoring and maintenance, production process failure prediction and diagnosis, etc. The injection molding machine control system was used to integrate industrial data of the machine and auxiliary production equipment. Industrial ethernet and industrial bus were used to interconnect all injection molding machines in the factory and perceive industrial data through the OPC (OLE for Process Control) protocol. The collected industrial data was transmitted from the edge layer to the cloud through the network connection layer based on the MQTT protocol (Message Queuing Telemetry Transport) [44-46]. Through industrial data modeling analysis and real-time interactive mapping, the production process dynamic control, fault diagnosis, process optimization, equipment monitoring, and other functions were realized. The management and control information could be accurately and quickly transferred to all levels of personnel in the company through various terminals such as PC, IOS, and Android. The flat and efficient management was realized through the application of the system.

The MES system with the multitenant SaaS was built based on the Spring Boot and Spring Could architecture, sharing database independently with the schema mode. The cloud platform realized dynamic data source switching and adopted the development model that separates front and back ends. The front-end development was based on the Vue and Element-UI framework to integrate IT and OT of the enterprise. Because the cloud platform was uniformly deployed on the cloud, so it could be operated directly by accessing the cloud, which reduced the cost of purchasing, constructing, and maintaining infrastructure, and it was also convenient and quick to upgrade and maintain the system.

5.3. Deconstruction and Application for the New Type of Industrial Collaborative Manufacturing Based on Big Data. Based on the sharing of external information resources by the enterprises and their upstream and downstream enterprises through the cloud platform, the industrial Internet cloud platform for injection molding industry was established (see Figure 11). Through this platform, combined with the application of big data analysis and artificial intelligence, it could carry out innovative services such as mold technical services, order capacity adjustment, production resource sharing, design and manufacturing collaboration, supplychain centralized trading, and talent training to reduce the cost of physical fitness of the enterprises and allocate resources to improve the overall efficiency of the industry. At last, intelligent production, networked collaboration, personalized customization, and service-oriented transformation for injection molding industry will ultimately be promoted.

The industrial Internet cloud platform of injection molding industry can be decomposed into the following five service subplatforms.

(1) Mold technology application subplatform: it provides the application services such as product design, mold design, injection process, CAD/CAM/CAE services, and testing services, which reduce the operating cost of the enterprises and promote the innovative development and collaborative innovation for various enterprises in the industry.

(2) Order adjustment and capacity allocation subplatform: it connects the equipment and manufacturing resources such as molding process, injection molding production, and equipment maintenance to form the cloud-based resource pool. Combining the platform promotion of new pieces of equipment and the sale service on behalf of the proposed agent, allocating production capacity on demand, and adjusting production undertaking, the capacity trading and order adjustment platform for equipment can be formed. Based on the equipment-sharing model, the transition from large-scale centralized production to distributed production can be realized.

(3) Product and mold design collaborative service subplatform: it communicates and connects terminal product $R \& D$ enterprises, their upstream supporting enterprises, and mold production enterprises. Through the unification of product and mold design standard, the collaborative service of product and mold design could be embedded in the network ecosystem to promote collaborative design and personalized customization of upstream and downstream enterprises in the industrial chain.

(4) Mold supply chain trading subplatform: the mold parts, plastic raw materials, and other materials of molding manufacturers and suppliers will be constituted in a cloud-based resource pool. Through the establishment of the strict selection platform for suppliers, centralized group purchase, and logistics of materials, recycling of sluggish materials can reduce the procurement cost for the enterprises.

(5) Technology training subplatform: through the integration of industry resources, a team of experts, engineers, and technical workers of injection molding industry can be established. The knowledge brain-bank based on industry knowledge, processes, cases, and experience can also be established and provides technical exchanges and training, enterprise technical consulting, and services.

On the cloud platform, the opening application services' interface can be used to expand continuously in accordance with the industry development needs and promote the formation of an intelligent format for injection molding industry. It can also be extended to other discrete manufacturing industries such as metal stamping industry.

\section{Application and Verification}

In this application example, the company is mainly engaged in the development, molding, and assembly of plastic molds for notebook computers and office equipment. It has two production factories located $70 \mathrm{~km}$ apart. There are many brands and models of injection molding machines such as Haitian, Chen Hsong, and Yizumi. It also applied ERP, 


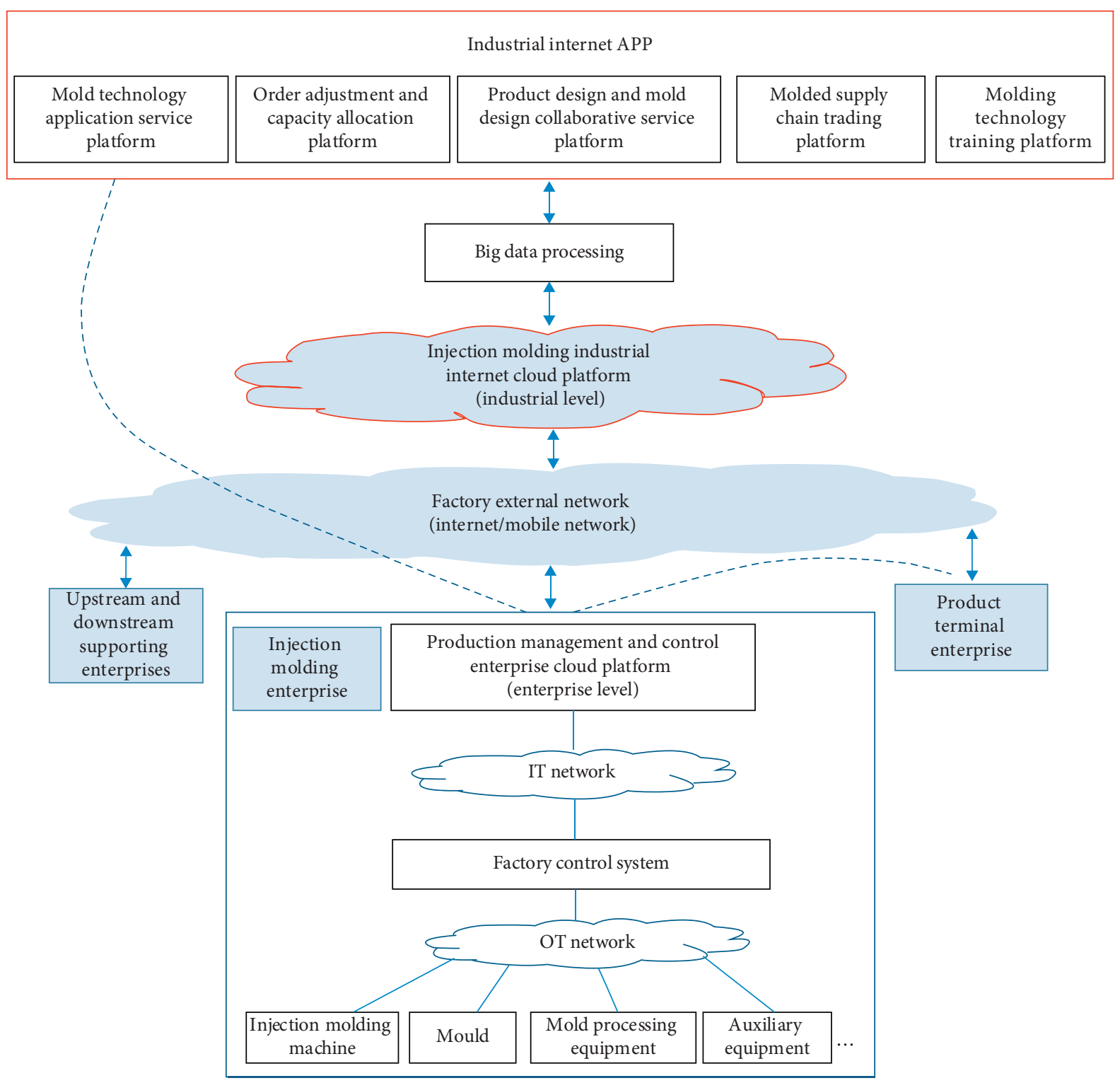

FIGURE 11: The industrial Internet cloud platform of injection molding industry.

industrial robots, other information systems, and automation equipments. However, there were still problems such as discrete storage of data, disconnection between production plans and workshop production, difficulty in real-time tracking and management of the production progress of orders and material usage, and cumbersome and inefficient personnel management. It was urgent to apply the MES production management and control cloud platform to realize the interconnection and centralized intelligent management and control of injection molding production equipment across regions and factories.

The industrial Internet system architecture constructed in conjunction with the production process of the company was implemented as follows (see Figure 12):

(1) Replace the control system of the original injection molding machine with the high-precision control system, which could improve the repetitive stability and product qualification rate, and realize the data collection and equipment networking of the process. The data acquisition module was used to obtain equipment state parameters for CNC machines.

(2) The network topology of the industrial Internet system is shown in Figure 13. The injection molding machines and $\mathrm{CNC}$ machines were connected to the workshop switch and edge server through industrial ethernet, and industrial data of these two production factories was transmitted to the cloud server of the company in real time based on the OPC and MQTT protocol.

(3) The virtual factory service system based on digital twin was established to perform logical processing on the industrial data of production process and 


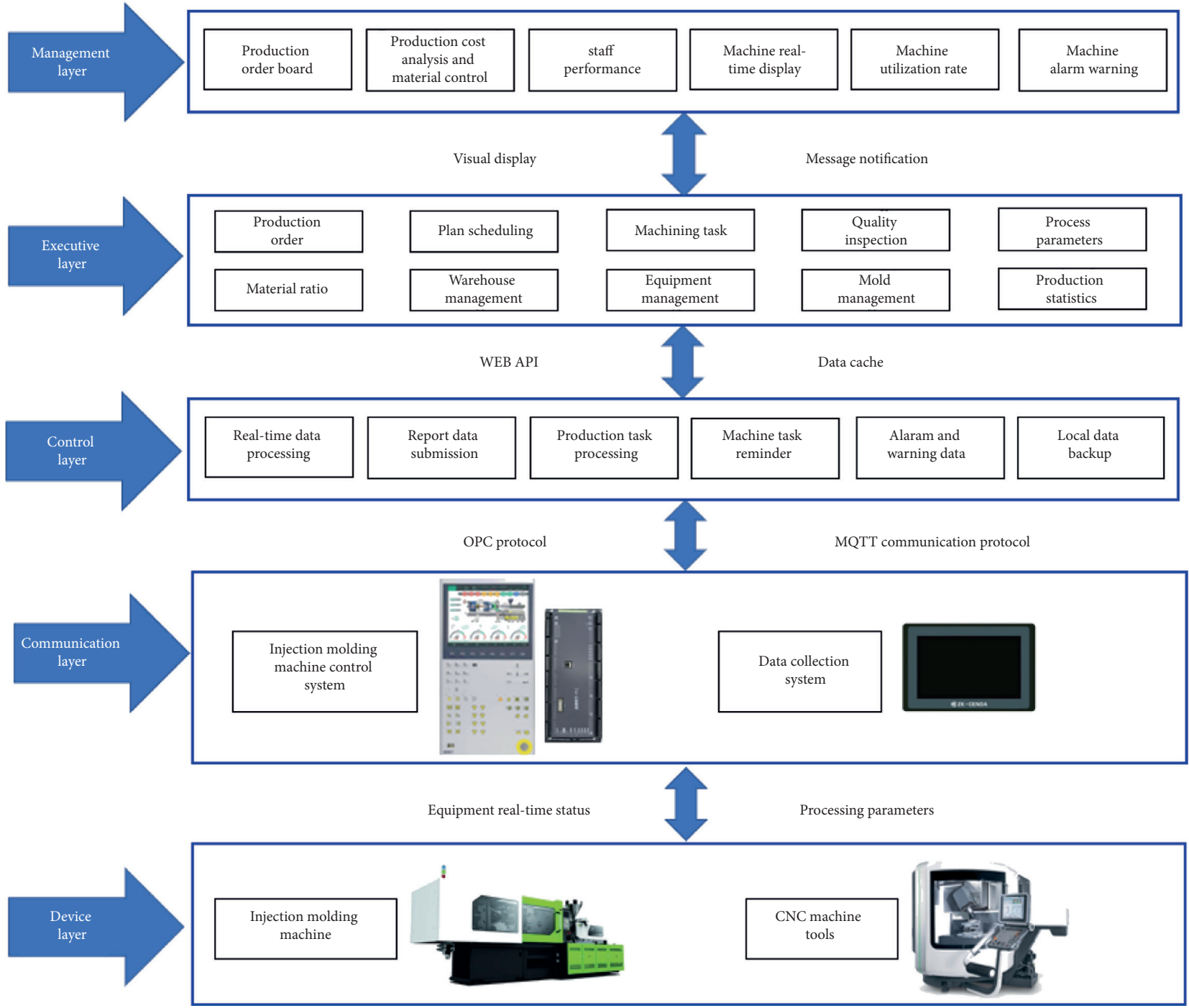

FIgURE 12: The architecture design of the industrial Internet system for the company.

simulation optimization through real-time collection of industrial data-driven models. It could realize automatic scheduling, real-time monitoring, fault prediction, and diagnosis of production plan and save the optimized data transmission to the digital twin database for subsequent data foundation and drive.

(4) Based on the SaaS, the MES for production management and control of enterprise cloud platform could view the production status, production plan, process parameters, production statistics, equipment maintenance, inventory management, cost accounting, and personnel performance of all workshops in real time through various terminals. It could also realize the interconnection and intercommunication of personnel and machines and things, which could solve the problems of equipment information islands and communication lag, realize the centralized management and control of cross-regional expansion of factories, and reduce the cost of redundant configuration of personnel.

The overview of the company's molding production management and control is shown (see Figure 14). Through nearly 18 months of project research, development, and implementation, the interconnection and centralized management of 137 injection molding machines in two factories had been achieved. The per capita production 


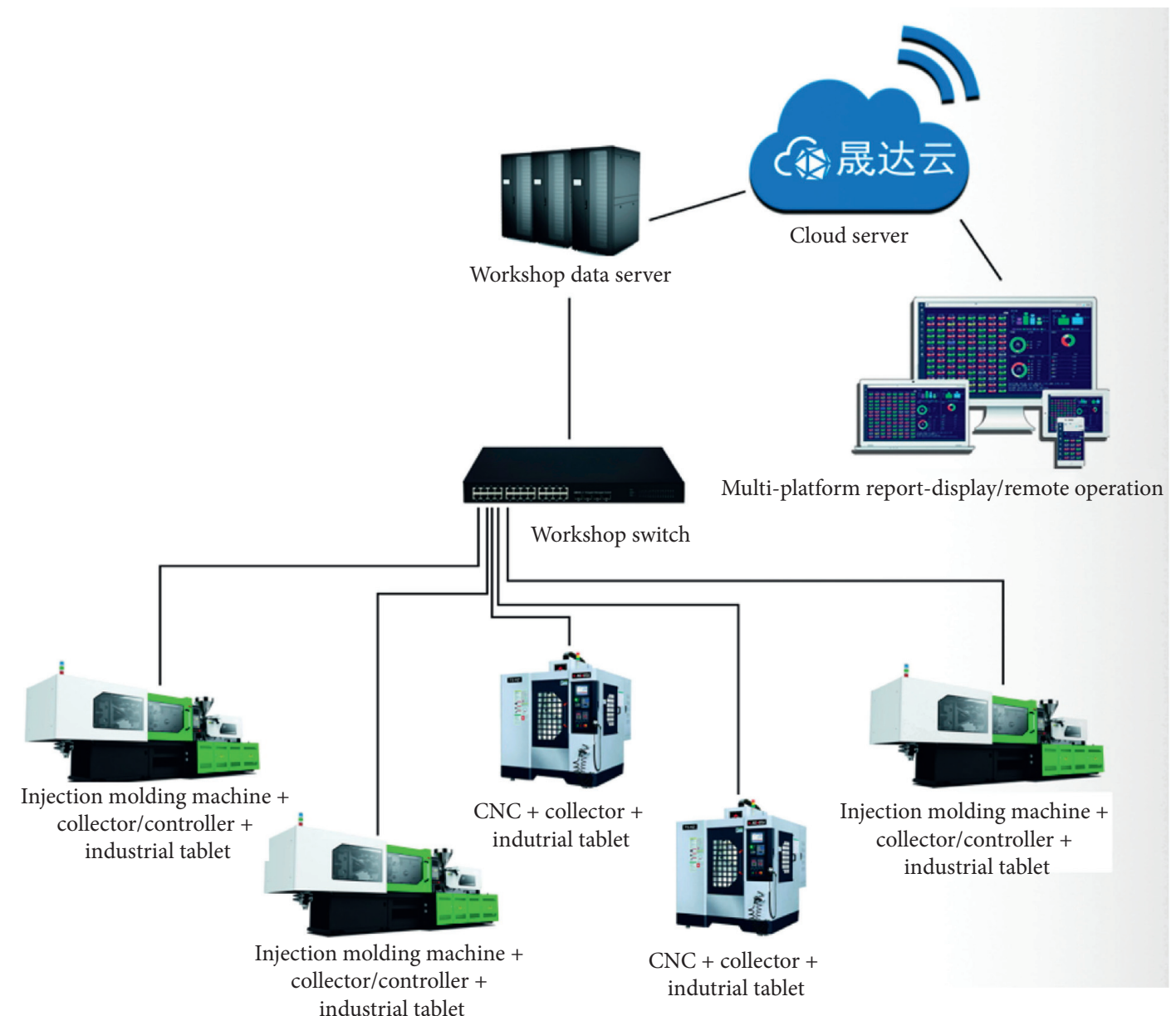

FIGURE 13: Network topology of the industrial Internet system for the company.

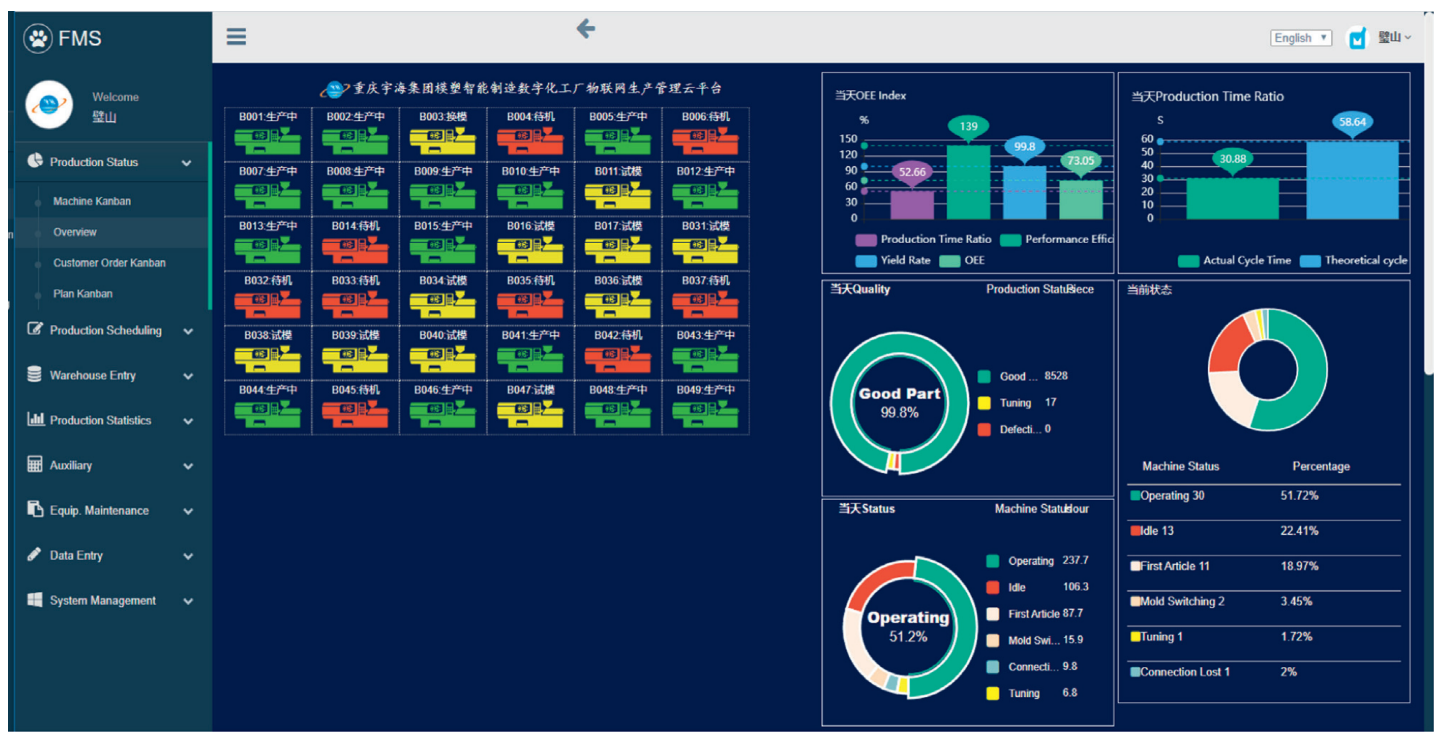

FIGURE 14: The real-time overview of the company with two cross-regional production factories. 
capacity increased by $4.8 \%$, yield rate increased by $1.6 \%$, total quality inspection increased by $8.9 \%$, and 32 staff were reduced, which created a certain benefit for the company.

\section{Conclusions}

In this paper, an industrial Internet system of injection molding industry was constructed, which provided the solution for smart factory based on digital twin, elaborated the implementation method, and verified the feasibility and effectiveness of the method through industrial application. The intelligence and informatization levels of China's injection molding enterprises are relatively low, so in-depth integration and innovative application with industrial Internet will be the key development direction in future. The application of industrial Internet will extend the value chain and life cycle of digital twin, and it will also promote the development of industrial Internet. Since China's injection molding enterprises are mainly small- and medium-sized, the establishment of the enterprise-level industrial Internet system could not only improve the production efficiency and management of these enterprises but also realize the centralized management and control of large enterprise groups across regions, reduce the duplication of personnel allocation, and increase operating efficiency. Through the industrial Internet cloud platform of injection molding industry, it will be helpful to promote the intelligent production, networked collaboration, personalized customization, and service-oriented transformation and form new industry formats with rich application values and broad development prospects.

\section{Data Availability}

No data were used to support this study.

\section{Conflicts of Interest}

The authors declare that they have no conflicts of interest regarding the publication of this paper.

\section{Acknowledgments}

This research work was supported by the Key-Area $R \& D$ Program of Guangdong Province (no. 2020B090925002), Joint Research Fund (no. U1813222) between National Natural Science Foundation of China (NSFC) and Shenzhen, and National Key R\&D Program of China (no. 2018YFB1309403).

\section{References}

[1] China Plastics Industry Association, "China plastics industry," China Plastics, vol. 31, no. 5, pp. 1-5, 2017.

[2] Z. Dong, "Development trend and market analysis of plastic products industry," Plastic Industry, vol. 44, no. 2, pp. 141144, 2016.

[3] G. Qian, "China's plastics processing industry adapts to a new era,” Plastics Engineering, vol. 70, no. 7, pp. 34-37, 2014.
[4] J. Zhou, "Intelligent manufacturing-the main direction of "Made in China 2025"," China Mechanical Engineering, vol. 26, no. 17, pp. 2273-2284, 2015.

[5] J.-Q. Li, F. R. Yu, G. Deng, C. Luo, Z. Ming, and Q. Yan, "Industrial internet: a survey on the enabling technologies, applications, and challenges," IEEE Communications Surveys \& Tutorials, vol. 19, no. 3, pp. 1504-1526, 2017.

[6] J. Yan and D. Kong, "Study on "industrial internet" and "industry 4.0"," Engineering Sciences, vol. 17, no. 7, pp. 141144, 2015.

[7] D. Dujovne, T. Watteyne, X. Vilajosana, and P. Thubert, "6TiSCH: deterministic IP-enabled industrial internet (of things)," IEEE Communications Magazine, vol. 52, no. 12, pp. 36-41, 2014.

[8] J. Posada, C. Toro, I. Barandiaran et al., "Visual computing as a key enabling technology for industrie 4.0 and industrial internet," IEEE Computer Graphics and Applications, vol. 35, no. 2, pp. 26-40, 2015.

[9] L. Jun and Q. Junjiang, "Evolution path, core capability construction and application of industrial internet platform," Science \& Technology Management Research, vol. 13, no. 2, pp. 182-186, 2019.

[10] J. Y. Zang, B. C. Wang, L. Meng et al., "Brief analysis on three basic paradigms of intelligent manufacturing," Chinese Journal of Engineering Science, vol. 20, no. 4, pp. 13-18, 2018.

[11] F. Tao and Q. Qi, "Make more digital twins," Nature, vol. 573, no. 7755, pp. 490-491, 2019.

[12] F. Tao, W. Liu, M. Zhang et al., "Five-dimension digital twin model and its ten applications," Computer Integrated Manufacturing Systems, vol. 25, no. 1, pp. 1-18, 2019.

[13] F. Tao, M. A. Xin, T. Hu et al., "Research on digital twin standard system," Computer Integrated Manufacturing Systems, vol. 25, no. 10, pp. 2405-2418, 2019.

[14] F. Tao, F. Sui, A. Liu et al., "Digital twin-driven product design framework," International Journal of Production Research, vol. 57, no. 12, pp. 3935-3953, 2019.

[15] F. Tao, J. Cheng, Q. Qi et al., "Digital twin-driven product design, manufacturing and service with big data," The International Journal of Advanced Manufacturing Technology, vol. 94, no. 12, pp. 3563-3576, 2018.

[16] Q. Qi, F. Tao, Y. Zuo, and D. Zhao, "Digital twin service towards smart manufacturing," Procedia CIRP, vol. 72, no. 8, pp. 237-242, 2018.

[17] F. Tao, W. Liu, J. Liu et al., "Digital twin and its potential application exploration," Computer Integrated Manufacturing Systems, vol. 24, no. 1, pp. 1-18, 2018.

[18] F. Tao, M. Zhang, J. Cheng et al., "Digital twin workshop: a new paradigm for future workshop," Computer Integrated Manufacturing Systems, vol. 23, no. 1, pp. 1-9, 2017.

[19] K. M. Alam and A. El Saddik, "C2PS: a digital twin architecture reference model for the cloud-based cyber-physical systems," IEEE Access, vol. 5, pp. 2050-2062, 2017.

[20] A. Vassiliev, V. Samarin, D. Raskin et al., "Designing the builtin microcontroller control systems of executive robotic devices using the digital twin technology," in Proceedings of 2019 International Conference on Information Management and Technology (ICIMTech), pp. 256-260, IEEE, Washington, D.C, USA, 2019.

[21] T. H.-J. Uhlemann, C. Lehmann, and R. Steinhilper, "The digital twin: realizing the cyber-physical production system for industry 4.0," Procedia Cirp, vol. 61, pp. 335-340, 2017.

[22] R. Rosen, G. von Wichert, G. Lo, and K. D. Bettenhausen, "About the importance of autonomy and digital twins for the 
future of manufacturing," IFAC-PapersOnLine, vol. 48, no. 3, pp. 567-572, 2015.

[23] B. Schleich, N. Anwer, L. Mathieu, and S. Wartzack, "Shaping the digital twin for design and production engineering," CIRP Annals, vol. 66, no. 1, pp. 141-144, 2017.

[24] A. Cerrone, J. Hochhalter, G. Heber et al., "On the effects of modeling as-manufactured geometry: toward digital twin," International Journal of Aerospace Engineering, vol. 2014, Article ID 439278, 10 pages, 2014.

[25] Z. Yu, Y. Sen, and C. Huanchong, "An application framework of digital twin and its case study," Journal of Ambient Intelligence and Humanized Computing, vol. 10, pp. 1141-1153, 2019.

[26] Q. Qi and F. Tao, "Digital twin and big data towards smart manufacturing and industry 4.0: 360 degree comparison," IEEE Access, vol. 6, pp. 3585-3593, 2018.

[27] H. Lu and Z. Yan, "Research and development of key technology for address space of OPC UA server," Dianli Zidonghua Shebei/Electric Power Automation Equipment, vol. 30, no. 7, pp. 109-113, 2010.

[28] D. Pampashree and A. Fakhruddin, "Design and implementation of SCADA based induction motor control," International Journal of Engineering Research \& Applications, vol. 4, pp. 5-18, 2014.

[29] S. Markus and K. Branko, "Service-oriented architecture design aspects of OPC UA for industrial applications," Lecture Notes in Engineering \& Computer Science, pp. 2175-2178, ISSN International Centre, Paris, France, 2009.

[30] Y. Yue-Xin, R. Gong-Chang, and Y. Wang, "Design of real time data acquisition system framework for production workshop based on OPC technology," MATEC Web of Conferences, vol. 128, Article ID 02014, 2017.

[31] J. Lu, Z. Cao, Z. Wang, and F. Gao, "A two-stage design of two-dimensional model predictive iterative learning control for nonrepetitive disturbance attenuation," Industrial \& Engineering Chemistry Research, vol. 54, no. 21, pp. 5683-5689, 2015.

[32] Z. Cao, Y. Yang, J. Lu, and F. Gao, "Two-time-dimensional model predictive control of weld line positioning in Bi-injection molding," Industrial \& Engineering Chemistry Research, vol. 54, no. 17, pp. 4795-4804, 2015.

[33] M. Gao, L. Sheng, D. Zhou, and F. Gao, "Iterative learning fault-tolerant control for networked batch processes with multirate sampling and quantization effects," Industrial \& Engineering Chemistry Research, vol. 56, no. 9, pp. 2515-2525, 2017.

[34] Z. Wang, R. Lu, F. Gao, and D. Liu, "An indirect data-driven method for trajectory tracking control of a class of nonlinear discrete-time systems," IEEE Transactions on Industrial Electronics, vol. 64, no. 5, pp. 4121-4129, 2017.

[35] Z. Cao, Y. Yang, H. Yi, and F. Gao, "Priori knowledge-based online batch-to-batch identification in a closed loop and an application to injection molding," Industrial \& Engineering Chemistry Research, vol. 55, no. 32, pp. 8818-8829, 2016.

[36] J. Shi, B. Yang, Z. Cao, H. Zhou, and Y. Yang, "Two-dimensional generalized predictive control (2D-GPC) scheme for the batch processes with two-dimensional (2D) dynamics," Multidimensional Systems and Signal Processing, vol. 26, no. 4, pp. 941-966, 2015.

[37] Y. Yang, B. Yang, S. Zhu, and X. Chen, "Online quality optimization of the injection molding process via digital image processing and model-free optimization," Journal of Materials Processing Technology, vol. 226, pp. 85-98, 2015.
[38] H. Y. Wong, K. T. Fung, and F. Gao, "Development of a transducer for in-line and through cycle monitoring of key process and quality variables in injection molding," Sensors and Actuators A: Physical, vol. 141, no. 2, pp. 712-722, 2008.

[39] V. Speranza, U. Vietri, and R. Pantani, "Monitoring of injection molding of thermoplastics: average solidification pressure as a key parameter for quality control," Macromolecular Research, vol. 19, no. 6, pp. 542-554, 2011.

[40] H. Gao, Y. Zhang, X. Zhou, and D. Li, "Intelligent methods for the process parameter determination of plastic injection molding," Frontiers of Mechanical Engineering, vol. 13, no. 1, pp. 85-95, 2018.

[41] H. Gao, Y. Zhang, X. Zhou et al., "Intelligent methods for the process parameter determination of plastic injection molding," Frontiers of Mechanical Engineering, vol. 13, pp. 1-11, 2017.

[42] F. X. You, X. Q. Zhao, and Y. L. You, "Production management and optimization in injection molding companies based on mes," in Proceedings of the 2017 2nd International Conference on Mechatronics, Control and Automation Engineering, pp. 253-259, Shenzhen, China, September 2017.

[43] L. Liu, H. DU, H. Wang et al., "Construction and application of digital twin system for production process in workshop," Computer Integrated Manufacturing Systems, vol. 25, no. 6, pp. 1536-1545, 2019.

[44] K. Tang, "Design and implementation of push notification system based on the MQTT protocol," in Proceedings of the International Conference on Information Science and Computer Applications (ISCA 2013), pp. 116-119, Changsha, China, November 2013.

[45] L. Feng, C. Pu, and J. Jun-Ying, "Application of WebSocket and MQTT in web real-time communication system," Computer Systems \& Applications, vol. 25, no. 5, pp. 28-33, 2016.

[46] H. Eslava, L. A. Rojas, and R. Pereira, "Implementation of machine-to-machine solutions using MQTT protocol in internet of things (IoT) environment to improve automation process for electrical distribution substations in Colombia," Journal of Power \& Energy Engineering, vol. 3, no. 4, pp. 92-96, 2015. 